\title{
Laser induced carbon nano-structures for planar antenna fabrication at microwave frequencies
}

\author{
David V. Thiel \\ Griffith School of Engineering \\ Griffith University, Nathan Qld 4111Australia \\ d.thiel@griffith.edu.au \\ Qin Li \\ Queensland Micro- \& Nanotechnology Centre \\ Griffith University, Nathan Qld 4111Australia \\ qin.li@griffith.edu.au
}

\author{
Xiangping Li, Min Gu \\ Centre for Micro-Photonics \\ Swinburne University of Technology, \\ Hawthorn Vic, 3122, Australia \\ xiangpingli@swin.edu.au \\ $\underline{\operatorname{mgu} @ \text { swin.edu.au }}$
}

\begin{abstract}
Printed antennas have environmental advantages over traditional copper/FR4 structures but the cost of silver remains an issue for mass production. Laser deposition of carbon nanostructures (grapheme) offer an even lower cost alternative. Three parallel lines (length $3 \mathrm{~mm}$, width $0.01 \mathrm{~mm}$ ) were fabricated on glass and the transmission coefficient was measured as a function of electric field orientation over the frequency range $8-10.6 \mathrm{GHz}$. The attenuation coefficient caused by parallel scattering was found to be linearly related $(r=0.7)$ to the relative length $l / \lambda_{0}$. The technology has potential applications in mass production of electronics and wireless sensor systems using the circuits in plastic technology as a low cost replacement for printed silver.
\end{abstract}

\section{INTRODUCTION}

Electronics is a polluting industry despite innovations designed to reduce some environmental impacts [1]. Problems associated with manufacture and recycling are partly due to the complex mixture of the materials (plastics, metals, glasses etc), but mainly due to and the end-of-life disposal. Given the competing demands of a reduced carbon foot-print and the consumer desires for new, sophisticated equipment, new technologies which address these issues are desirable. Circuits in plastic (CiP) [2] is one such technology currently undergoing trials. Recent reports [3] have indicated that recycled plastic and bioplastics are viable alternatives to fossil fuel based new plastic materials. Should these environmentally friendly materials become widely accepted in the industry, there are still problems associated with the conducting materials used to form the circuits and antennas. Ink-jet and screen printed colloidal silver is expensive and, when circuits are placed in landfills, they are polluting. Laser deposition of carbon films might prove a viable alternative, both from a cost and environmental perspective.

Among various carbon nanomaterials, graphene currently attracts most attention, owing to its extraordinary electrical and mechanical properties as well as the continuous improvement in its synthesis method and reduced cost. One recently published method creates graphene-oxide (GO) like thin carbon "nanosheets" using readily available materials glycerol, sulfuric acid and melamine [4]. It should be noted that the immediate product of wet chemistry synthesis is always GO, which is not conductive and requires a subsequent reduction process to turn GO into graphene. Laser irradiation has proven to be an effective method in reducing GO [5]. The contrast in conductivity between GO and graphene allows the laser to directly create graphene nanostructures on any substrate which has been deposited with GO.

Conductivity measurements on nanostructures at microwave frequencies are difficult because of such effects as the electrode potentials, stray capacitance and the difficulties in positioning the probes. An alternative electromagnetic method has been used to determine the properties of carbon fiber reinforced laminates with [6], anisotropic substrates [7] and printed frequency selective surfaces [8].

\section{THEORY}

A conductive strip sandwiched between a dielectric substrate and a dielectric superstrate has an effective length influenced by the material relative permittivity $\varepsilon_{r}$ and the thicknesses of the two layers. For microscope slide glass the relative permittivity $\varepsilon_{r} \cong 2.3$. There is no exact formulation to determine the effective permittivity $\varepsilon_{\text {eff }}$ which can be used to calculate the wavelength $\lambda$, along the conducting line. This can be written as

$$
\lambda=\frac{\lambda_{0}}{\sqrt{\varepsilon_{e f f}}} \quad \text { and } 1<\varepsilon_{e f f}<\varepsilon_{r}
$$

where $\lambda_{0}$ is the free space wavelength of the radiation.

For the three parallel conducting lines the effective area at $10 \mathrm{GHz}$ is approximately $1.1 \times 10^{4} \mathrm{~m}^{2}$ which is approximately $46 \%$ of the cross sectional area of the waveguide. If the electric field is perpendicular to the conductors the effective aperture approaches zero. A measurement of the received signal when the electric field is aligned will be significantly different to when orthogonally aligned. The electric field ratio is independent of the glass and residual GO. 


\section{EXPERIMENTAL METHOD}

\section{A. Carbon line fabrication}

GO flakes were drop cast on a glass cover slip and dried at room temperature. A fs pulsed laser beam at the wavelength of $800 \mathrm{~nm}$ (repetition rate of $1 \mathrm{kHz}$ and a pulse width of $100 \mathrm{fs}$ ) was employed for the photoreduction. The $0.2 \mathrm{~mW}$ laser beam was focused by an objective lens with the numerical aperture of 0.4 . The reduced graphene lines were realized by lateral translation of the sample across the focal plane at $10 \mu \mathrm{m} / \mathrm{s}$. Three parallel lines were fabricated (length $3 \mathrm{~mm}$, width 0.05 $\mathrm{mm}$, separation $1 \mathrm{~mm}$ ). The samples were mounted on a glass microscope cover slip and placed on a microscope slide (75 $\mathrm{mm} \times 25 \mathrm{~mm} \times 1 \mathrm{~mm}$ ).

\section{B. Scattering measurements}

An X-band pyramidal horn was placed $130 \mathrm{~mm}$ from an open rectangular waveguide (internal dimensions $24 \mathrm{~mm} \times 10$ $\mathrm{mm}$ ). The horn was driven by a microwave synthesizer over the frequency range $8-10.6 \mathrm{GHz}$ using the $\mathrm{TE}_{01}$ propagation mode. The received signal was measured using a Marconi 28B frequency counter with frequency and signal level readout. The sample under test was placed against the flange in front of the open waveguide and centered in both dimensions where the electric field strength a maximum for the $\mathrm{TE}_{01}$ mode.

The received signal level $(\mathrm{dB})$ was recorded as a function of frequency. The system was calibrated in air. The sample was placed in the center of the receiving aperture with the lines oriented parallel $\left(E_{\|}\right)$and perpendicular to the $\left(E_{\perp}\right)$ field. The received parallel power $(\mathrm{dBm})$ was compared to the received perpendicular power.

\section{EXPERIMENTAL RESULTS}

The received ratio of the electric field strength $E_{\|} / E_{\perp}$ is shown in fig. 1 as a function of the effective length (relative to the free space wavelength $\lambda_{0}$. The relationship is approximately linear (Pearson correlation coefficient $r=-0.707$ ) indicating that the scattering is linearly related to the electrical length of the three strips.

\section{DISCUSSION AND CONCLUSIONS}

The scatter in the data is caused by waveguide resonances, and positional uncetainties of the sample. As the effective permittivity of the glass slide and cover slip is unknown, the results in Fig. 1 will be shifted to the right for $\lambda<\lambda_{0}$. At low frequencies there is very little scattering but as the electical length of the conductors increases, the scattering increases and the attenuation of the signal increases. The effect of the conductivity of the lines is low providing a minimum threshold has been met [8].

The use of laser defined carbon nanosheets offers an environmentally friendly, flexible, low-cost, solution to circuits in plastic manufacturing and the fabrication of planar antennas on a wide variety of substrate materials. Laser patterning of planar antennas such as meander lines will result in substantial cost savings relative to the current silver ink processes.

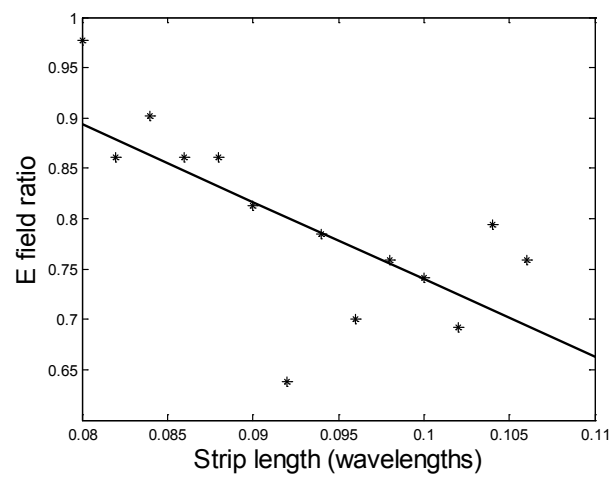

Fig. 1. Electric field strength ratio $\mathrm{E}_{\|} / \mathrm{E}_{\perp}$ measured as a function of the effective length of the three parallel conducting lines. The linear fit has a correlation coefficient of $r=-0.70$.

\section{ACKNOWLEDGEMENT}

The research team thanks the Australian Research Council for its support (FL100100099, DP110101422, DP130102098).

\section{REFERENCES}

[1] S. Herat, "Sustainable management of electornic waste (e-waste)," Clean - Soil Air Water, vol. 35, pp. 305-310, 2007.

[2] M. Neeli, S. Raj and D.V. Thiel, "Plastic circuit reliability and design for recycling," $11^{\text {th }}$ EPTC, Taipai, Taiwan, pp. 858-862, Dec. 2009.

[3] M. Neeli, M. Okine and D.V. Thiel, "Lifecycle analysis of plastics in South-East Queensland: recycled plastics versus bioplastics in electronics," Nanotech, vol. 3, pp. 761-764, 2013.

[4] W. Wang, S. Chakrabarti, Z. Chen, Z. Yan, M.O. Tade, J. Zou, and Q. $\mathrm{Li}$, "A novel bottom-up solvothermal systhesis of carbon nanosheets," J. Materials Chem. A., DOI:10.1039/C3TA13593D.

[5] X. Li, Q. Zhang, X. Chen \& M. Gu, Giant refractive-index modulation by two-photon reduction of fluorescent graphene oxides for multimode optical recording, Scientific Reports, 3, DOI 10.1038/srep02819, 2013.

[6] Galehdar, W.S.T. Rowe, K. Ghorbani, P.J. Callus, S. John and C.H. Wang, "The effect of ply orientation on the performance of antennas in or on carbon fiber composites," PIERS vol. 116, pp. 123-126, 2011.

[7] D.V. Thiel, M. Kanesan, and W. Hall, "Printed antennas on fiber reinforced anisotropic polymeric substrates," IEEE APS/URSI Sym. Memphis, TE., July 2014. (submitted).

[8] A.A. Dewani, M. Kanesan, D.V. Thiel, S.G. O'Keefe and M. Vatankhah Varnoosfaderani, "Screen printed frequency selective surfaces for room isolation in buildings," iWAT, Sydney 2014 (in press)

[9] M. Shahpari and D.V. Thiel "The performance of parasitic element in Yagi-Uda antennas at low conductivity," PIERS (submitted) October 2013. 